\title{
Análisis de riesgo en la evaluación de alternativas de inversión utilizando Crystal Ball ${ }^{\circledR}$
}

Recepción: Febrero de 2007 / Aceptación: Mayo de 2007

(1) Javier Del Carpio Gallegos (2) Roberto Eyzaguirre Tejada

\begin{abstract}
RESUMEN
El presente artículo muestra cómo el uso del software Crystal Ball $\circledast$, incorporado en una hoja de cálculo, puede ayudar a tomar mejores decisiones de inversión aplicando la técnica de simulación Monte Carlo.

Palabras Clave: Riesgo, Análisis de Inversión, Crystal Ball@

CAPITAL BUdGETING RISK ANALYSIS APPLYING CRISTAL BALL ${ }^{\circledR}$

ABSTRACT

This article shows how to apply Crystal Ball@ software to make better investment decisions applying Monte Carlo simulation technique.
\end{abstract}

Key Words: Risk, Capital Budgeting, Crystal Ball@

\section{INTRODUCCIÓN}

La globalización de los negocios ha llevado a que la toma de decisiones sea más complicada. En tal sentido los administradores financieros han concentrado su atención en el uso de herramientas que les permita plantear diferentes escenarios, y el uso de las técnicas conocidas de simulación, como es el caso del método de Monte Carlo.

La revisión de la literatura pertinente [1, 2, 3, 5] ilustra profusamente cómo la técnica Monte Carlo ayuda a enfrentar el análisis de riesgo en la toma de decisiones de evaluaciones de alternativas de inversión.

El Dr. Johnathan Mun en su libro Applied Risk Analysis señala la importancia del riesgo en la toma de decisiones [6], y precisa la diferencia entre riesgo e incertidumbre: "Los conceptos de riesgo e incertidumbre están relacionados pero son muy diferentes. La incertidumbre involucra variables que están constantemente cambiando, mientras que el riesgo involucra solamente las variables inciertas que afectan o impactan directamente el resultado del sistema."

\section{ANÁLISIS DE RIESGO}

Es conveniente tener en cuenta que el riesgo es la probabilidad de tener éxito o fracaso, cuando se toma la decisión de llevar a cabo un proyecto, por tal motivo se hace necesario realizar una evaluación del riesgo que se está asumiendo, y para tal efecto mediante la aplicación del método del análisis de riesgo, el cual es un método de tipo probabilístico, que considera que se puede asociar las variables con una distribución de probabilidades.

\section{Distribución de probabilidad}

La distribución de probabilidad puede ser discreta o continua. Un ejemplo de distribución discreta es cuando se asume que el Valor Actual Neto (VAN) puede tomar distintos valores, y cada uno de ellos asociados con una probabilidad determinada, tal como se muestra en el cuadro 1.

Cuadro 1. Distribución del VAN

\begin{tabular}{|c|c|}
\hline VAN(S/) & Probabilidad \\
\hline 500 & 0,50 \\
\hline 600 & 0,30 \\
\hline 700 & 0,20 \\
\hline Sumatoria & $\mathbf{1 , 0 0}$ \\
\hline
\end{tabular}

Fuente: Elaboración propia

(1) Magíster. Profesor del Departamento de Producción y Gestión Industrial, UNMSM

E-mail: jdelcarpiog@unmsm.edu.pe

(2) Ingeniero Industrial. Profesor del Departamento de Ingeniería de Sistemas e Informática, UNMSM

E-mail: reyzaguirret@unmsm.edu.pe 
Figura 1. Distribución Normal del VAN

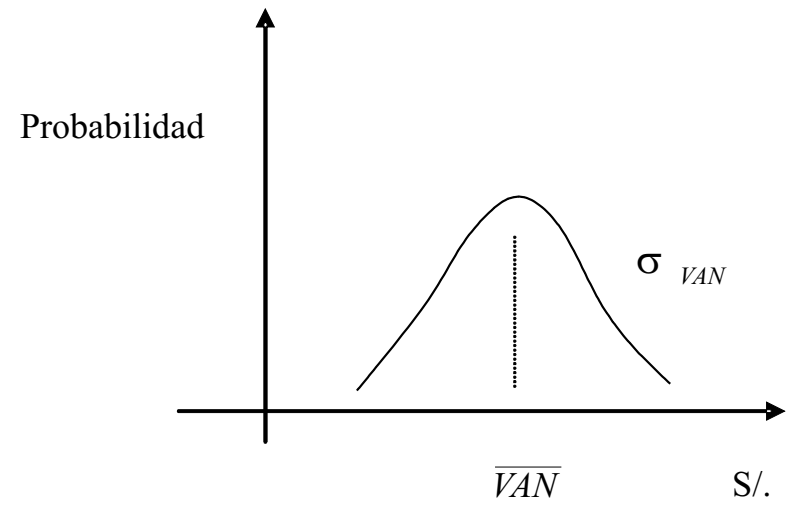

Fuente: Elaboración propia

En forma similar se puede asumir que el VAN sigue una distribución de probabilidades de tipo normal con media $\overline{V A N}$, y desviación estándar $\sigma_{V A N}$, como se muestra en la figura 1.

\section{EJEMPLODEAPLICACIÓN}

\section{Planteamiento del problema}

La empresa $A B C$ tiene que llevar a cabo un proyecto que requiere de una inversión de $S / .1000$ en activos fijos (los cuales tienen una vida útil de 5 años), y adicionalmente S/ 200 como capital de trabajo. La demanda estimada se muestra en el cuadro 2.

El precio unitario es de $S / 12$, los costos variables totales representan el $25 \%$ de las ventas, y los costos fijos anuales que no incluyen la depreciación son de $\mathrm{S} / \mathrm{100}$. Se considera que el horizonte del proyecto es de 4 años, y se asume que la tasa del impuesto a la renta es de $30 \%$. Se ha considerado que el pronóstico de las ventas sigue una distribución triangular como se detalla en el cuadro 3.

De manera similar, en el cuadro 4, se ha considerado que los costos variables siguen una distribución triangular.

Elaboración de los estados de ganancias y pérdidas para cada uno de los cuatro períodos

La figura 2 muestra el ingreso de los datos en la hoja de cálculo electrónica EXCEL que tiene las funciones adicionales del software Crystal Ball囚.

\section{Elaboración del Flujo de Fondos Económico,}

En la figura 3 se aprecia el Flujo de Fondos y la determinación del Valor Actual Neto Económico que asciende a S/. 1123,98, este cálculo se ha efectuado considerando los valores de las ventas y de los costos variables totales en el escenario normal. En forma similar se ha calculado la Tasa Interna de Retorno Económico obteniendo el resultado de 46,91\%.

\section{Pronóstico del Valor Actual Neto Económico}

Siguiendo lo recomendado por Lawrence I. Goldman [4] se utiliza la función pronóstico (Forecast) del VANE y se obtiene una gráfica como se muestra en la Figura 4 , después de haber realizado 1000 iteraciones se encuentra la distribución del VANE.

Determinación de la probabilidad que el VAN sea mayor que cero.

Habiendo efectuado la simulación correspondiente se puede determinar la probabilidad que el VANE sea mayor que 1100, y la Figura 5 muestra que este valor es de $97,63 \%$.

Pronóstico de la tasa interna de retorno económico

En forma similar la Figura 6 muestra la distribución de la tasa interna de retorno después de haber efectuado 1000 iteraciones.

\section{Determinación de la probabilidad que la TIR sea} mayor que $45 \%$.

La Figura 7 muestra la probabilidad que la tasa interna de retorno económico sea mayor que $45 \%$, en este caso el resultado es de $83.88 \%$.

Cuadro 2. Demanda estimada en unidades

\begin{tabular}{|l|c|c|c|c|}
\hline Período & 1 & 2 & 3 & 4 \\
\hline Ventas (Unidades) & 100 & 110 & 110 & 120 \\
\hline
\end{tabular}

Fuente: Elaboración propia

Cuadro 3. Pronóstico de ventas (Unidades)

\begin{tabular}{|c|c|c|c|}
\hline Periodo & Pesimista & Normal & Optimista \\
\hline 1 & 90 & 100 & 110 \\
\hline 2 & 99 & 110 & 121 \\
\hline 3 & 99 & 110 & 121 \\
\hline 4 & 108 & 120 & 132 \\
\hline
\end{tabular}

Fuente: Elaboración propia

Cuadro 4. Costos Variables en Nuevos Soles

\begin{tabular}{|c|c|c|c|}
\hline Periodo & Pesimista & Normal & Optimista \\
\hline 1 & 270 & 300 & 330 \\
\hline 2 & 297 & 330 & 363 \\
\hline 3 & 297 & 330 & 363 \\
\hline 4 & 324 & 360 & 396 \\
\hline
\end{tabular}

Fuente: Elaboración propia 
Figura 2. Estado de Ganancias y Pérdidas (En el escenario normal aplicando el software Crystal Ball(®

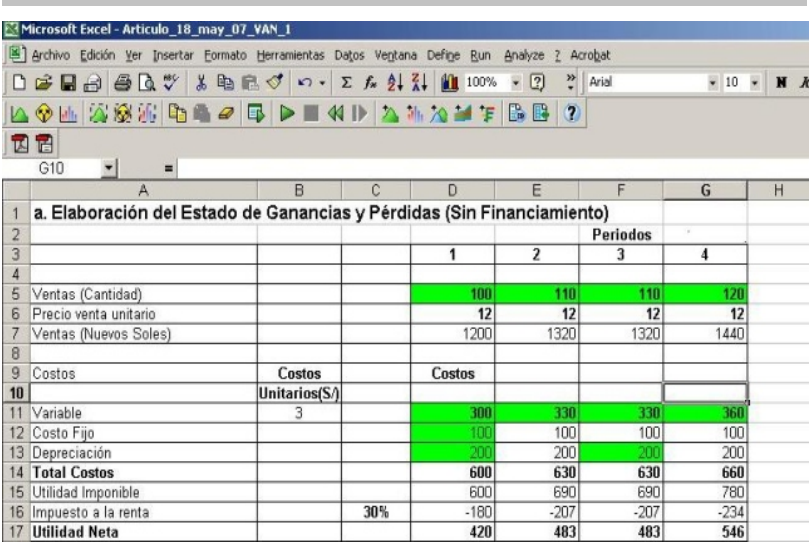

Fuente: Elaboración propia

Figura 4. Pronóstico del VANE aplicando el software Crystal Ball@

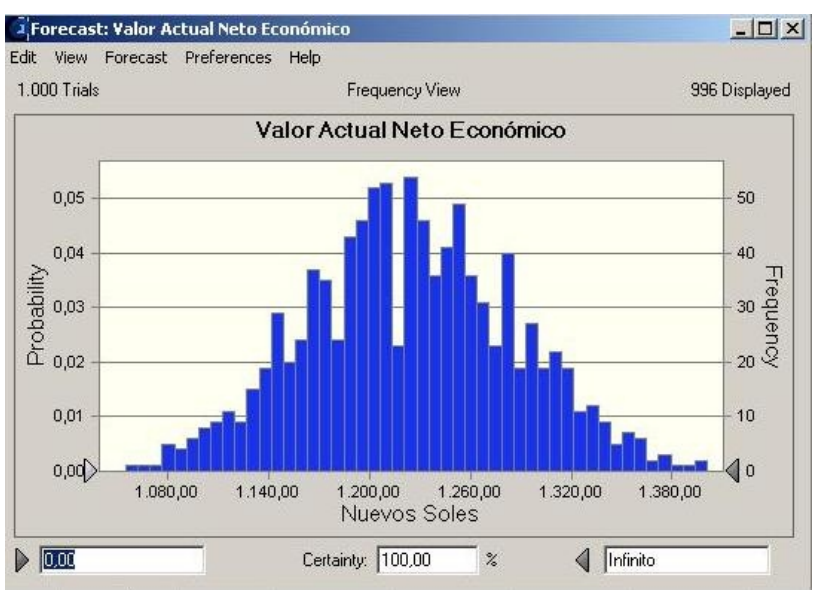

Fuente: Elaboración propia

Figura 6. Pronóstico de la TIRE aplicando el software Crystal Ball@

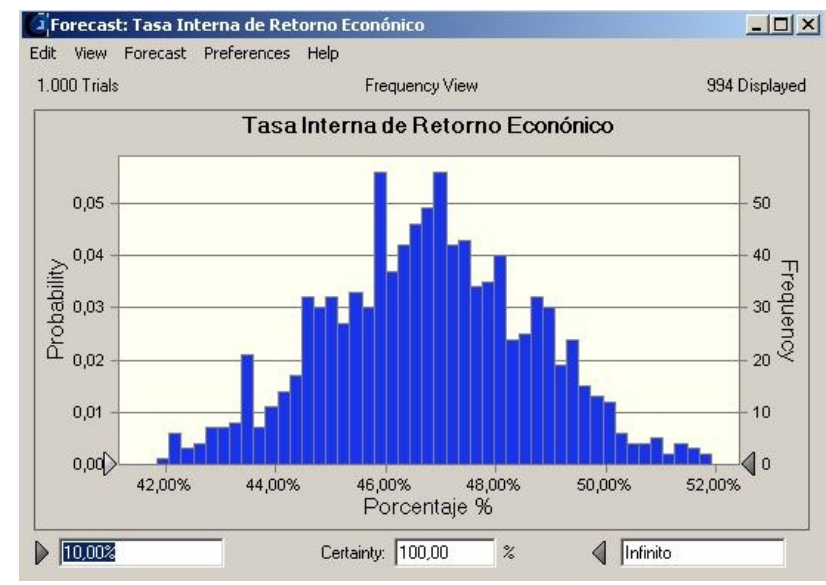

Fuente: Elaboración propia
Figura 3. Determinación del VANE y el TIRE aplicando el software Crystal Ball@

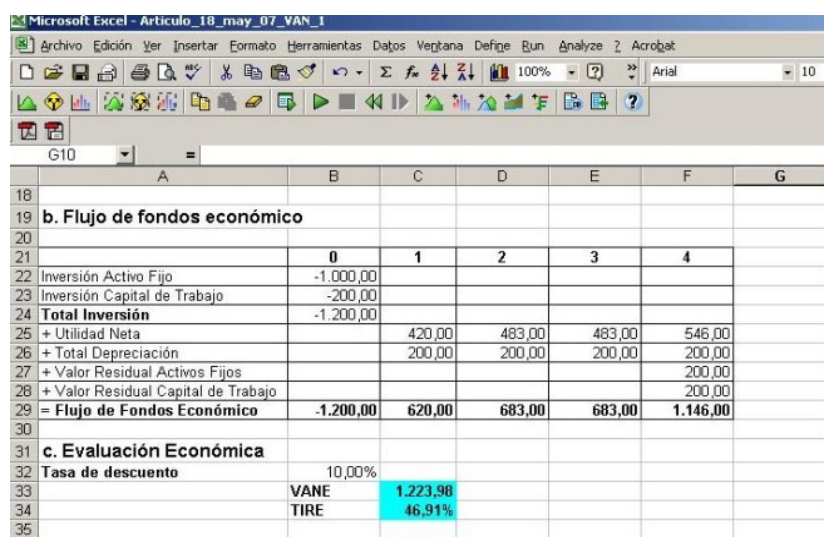

Fuente: Elaboración propia

Figura 5. Probabilidad que VANE sea mayor que cero aplicando el software Crystal Ball@

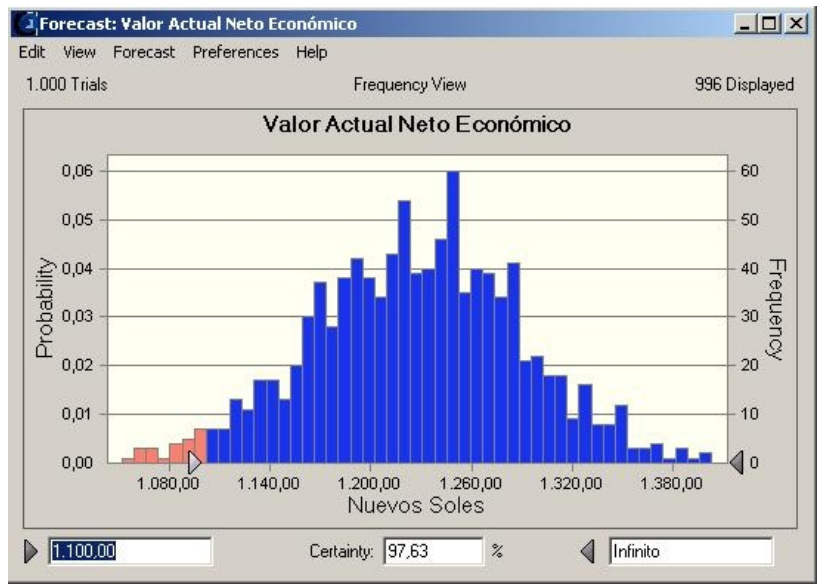

Fuente: Elaboración propia

Figura 7. Probabilidad que TIRE sea mayor que $45 \%$ aplicando el software Crystal Ball@

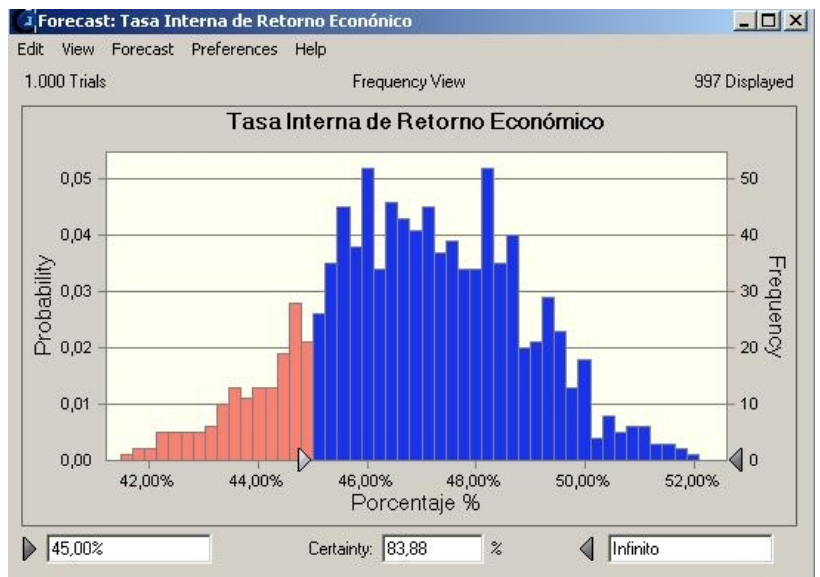

Fuente: Elaboración propia 
Figura 8. Análisis de sensibilidad del VANE aplicando el software Crystal Ball@

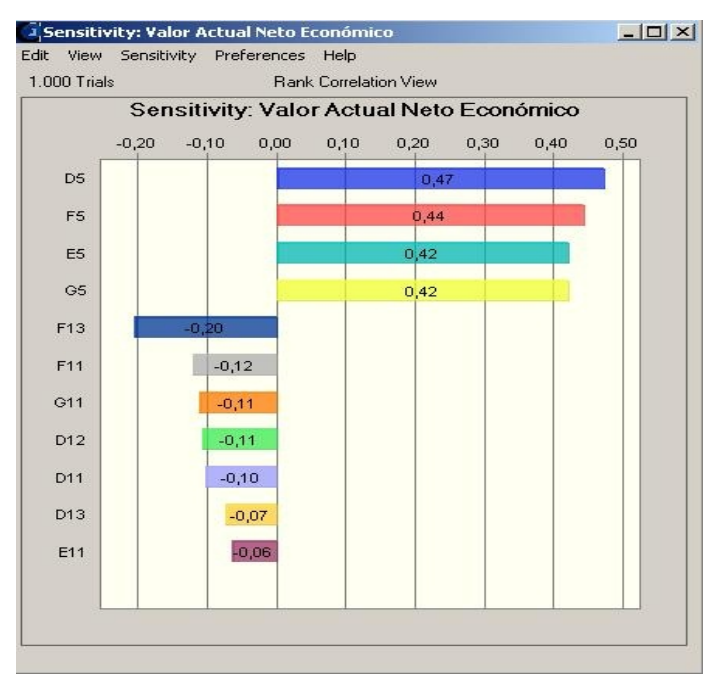

Fuente: Elaboración propia

\section{Análisis de sensibilidad del Valor Actual Neto Económico (VANE).}

La Figura 8 muestra el análisis de sensibilidad del VANE señalando el ranking de la correlación de las distintas variables que se han considerado, como es el caso de la demanda estimada en unidades y el costo variable unitario, y que pueden tener una mayor o menor influencia en la determinación del VANE.

En el cuadro 5 se muestra el ranking de las correlaciones positivas o negativas que tendrían un impacto sobre la determinación del Valor Actual Neto Económico. Es decir, en el caso de la demanda en unidades del año 1, tiene una correlación positiva del $47 \%$ con el Valor Actual Neto Económico. En tal sentido un incremento de la demanda en unidades en el año 1 , tendrá un impacto del $47 \%$ sobre el Valor Actual Neto Económico. Por otro lado, la aplicación de esta opción permite identificar las variables que tienen un mayor impacto positivo o negativo sobre la variable que se va a pronosticar.

\section{CONCLUSIONES}

En el desarrollo del ejemplo de aplicación se muestra que la técnica de simulación de Monte Carlo aporta significativamente en el análisis de inversión bajo condiciones de riesgo. Pues el método determinístico produce un solo valor como es el caso del VANE igual a S/.1.223,98. En tanto que la aplicación de Monte Carlo indica una distribución de probabilidades del VANE, determinando que la probabilidad que el VANE sea mayor que $S / .1 .100,00$ se estima en $97,63 \%$.

\section{Cuadro 5. Ranking de correlación}

\begin{tabular}{|c|c|c|}
\hline Variable & Descripción & Correlación \\
\hline D5 & Demanda estimada unidades año 1 & $+0,47$ \\
\hline F5 & Demanda estimada unidades año 3 & $+0,44$ \\
\hline E5 & Demanda estimada unidades año 2 & $+0,42$ \\
\hline G5 & Demanda estimada unidades año 4 & $+0,42$ \\
\hline F13 & Depreciación año 3 (S/.) & $-0,20$ \\
\hline F11 & Costo variable año 3 (S/.) & $-0,12$ \\
\hline D12 & Costo fijo año 1 (S/.) & $-0,11$ \\
\hline D11 & Costo variable año 1 (S/.) & -0.10 \\
\hline D13 & Depreciación año 1 (S/.) & -0.07 \\
\hline F15 & Utilidad imponible año 3 (S/.) & $-0,06$ \\
\hline
\end{tabular}

Fuente: Elaboración propia

La ayuda de un software como Cristal Ball @ simplifica el análisis de riesgo en las decisiones de inversión. De esta manera, los modelos determinísticos pueden ser enriquecidos con el uso de aplicaciones informáticas que permitirán enfrentar con mayor éxito situaciones tan cambiantes como las vigentes.

\section{REFERENCIAS BIBLIOGRÁFICAS}

1. Berza Garmendia, J. (2004).Herramientas de Excel para el Análisis y Valoración de Proyectos de Inversión. Revista de Dirección y Administración de Empresas. Número 11, marzo 2004: 51-70.

2. Castelo Joaquín, D. (2005). Capital Budgeting Exercise using Simulation. III in o is State University. En: http://www.ilstu.edu.

(Visitado: 09-11-06).

3. Dapena, J. (2005). Simulación aplicada al Análisis de Proyectos de Inversión Técnica SAAPI. Temas de Management, Volumen III: 20-26

4. Goldman, L. (2002). Crystal Ball Professional Introductory Tutorial. Proceedings of the 2002 Winter Simulation Conference.

5. Hyde, J. and Engel, P. (2002). Investing in a Robotic Milking System: A Monte Carlo Simulation Analysis. Journal Dairy Science. 85: 2207-2214.

6. Mun, Johnathan. (2004). Applied Risk Analysis. John Wiley \& Sons, Inc. U.S.A. 